medRxiv preprint doi: https://doi.org/10.1101/2020.04.28.20075523; this version posted May 4, 2020. The copyright holder for this preprint (which was not certified by peer review) is the author/funder, who has granted medRxiv a license to display the preprint in perpetuity.

All rights reserved. No reuse allowed without permission.

\title{
Detection of SARS-CoV-2 in Human Breast Milk
}

\author{
Rüdiger Groß ${ }^{1}$, Carina Conzelmann ${ }^{1}$, Janis A. Müller ${ }^{1}$, Steffen Stenger ${ }^{2}$, Karin Steinhart ${ }^{3}$, Frank \\ Kirchhoff $^{1}$, Jan Münch ${ }^{*}$ \\ ${ }^{1}$ Institute of Molecular Virology, Ulm University Medical Center, Ulm, Germany \\ ${ }^{2}$ Institute for Microbiology and Hygiene, Ulm University Medical Center, Ulm, Germany \\ ${ }^{3}$ Administrative District Heidenheim, Public Health Office, Heidenheim, Germany \\ *Corresponding author: jan.muench@uni-ulm.de
}

It is currently unclear whether SARS-CoV-2 (CoV-2) may be shed into breast milk and transmitted through breastfeeding. Recent reviews and research articles reported no evidence for CoV-2 in human breast milk but the sample size was small. ${ }^{1-3}$

Here, we examined milk samples from two CoV-2 infected nursing mothers (M1 and M2). An overview on the clinical data and time course of infection is provided in Fig. 1A. We determined CoV-2 viral loads using RT-qPCR for CoV-2 N and -ORF1b ${ }^{4}$ (Fig. S1) in whole milk and skim milk obtained after removal of the lipid fraction. All four milk samples obtained from M1 from day 12 to 14 tested negative (Fig. 1B). In contrast, CoV-2 RNA was detected in milk provided by M2 on days 10 (left and right breast), 12, 13 and 14, while a later sample from day 25 was negative (Fig. 1A). Peak levels were detected on day 12 with Ct values for CoV-2 N of 29.8 and 30.4 in whole milk and skim milk, respectively (Fig. 1B, top), corresponding to viral genome copy numbers of $1.32 \times 10^{5}$ and $9.48 \times 10^{4}$ per ml (mean of both isolations, Fig. 1B, bottom). Since milk components may affect RNA isolation and quantification, viral RNA recovery rates upon spiking milk with serial dilutions of a CoV-2 stock were determined. We observed an up to $89.2 \%$ reduced recovery rate in whole milk and $51.5 \%$ in skim milk (Fig. S2) suggesting that the actual viral loads in whole milk of M2 may be even higher (Fig. 1C).

We detected CoV-2 RNA in milk samples collected over a period of 4 days. Based on specimen handling, internal controls, and negative testing of M1 milk, contamination can be excluded. Detection of viral RNA in milk of M2 coincided with mild COVID-19 symptoms and a CoV-2 positive diagnostic testing of the newborn (N2) (Fig. 1A). M2 was wearing a surgical mask since the onset of symptoms and followed safety precautions during handling or feeding the neonate, including proper hand and breast disinfection, strict washing and sterilization of milk pumps and tubes. However, whether N2 was infected by breastfeeding or other modes of transmission remains unclear. Further studies of milk samples from lactating women and possible virus transmission via breastfeeding are needed to develop recommendations on whether mothers with COVID-19 should breastfeed.

Authors' Contributions:

R.G., C.C. and J.A.M. contributed equally to this study. R.G. performed RNA extraction, RT-qPCR analysis and evaluated data. C.C. and J.A.M. generated virus stock handled milk samples and prepared them for RNA extraction. S.S. supervised BSL3 work and helped in writing. K.S. organized samples and was involved in study design. F.K. and J.M. supervised the project and wrote the article.

Ethics statement:

Both mothers were informed about the study and gave informed consent. Ethical approval for this study was waived by the Ethics Committee of Ulm University because this study is case report and all samples were anonymized.

NOTE: This preprint reports new research that has not been certified by peer review and should not be used to guide clinical practice. 
A

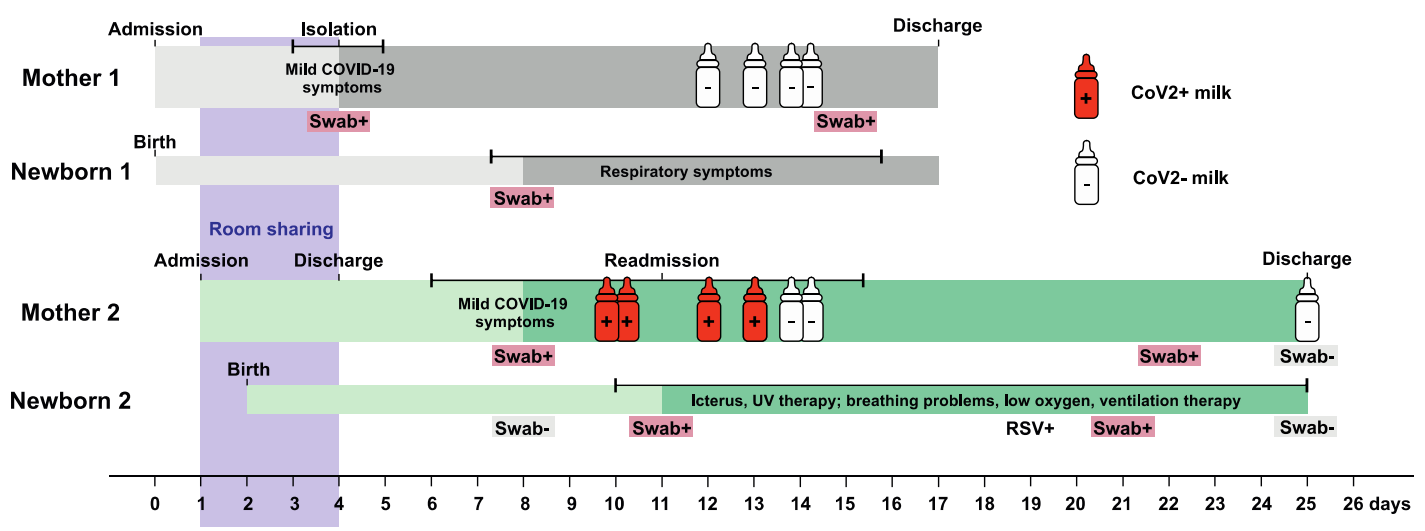

B
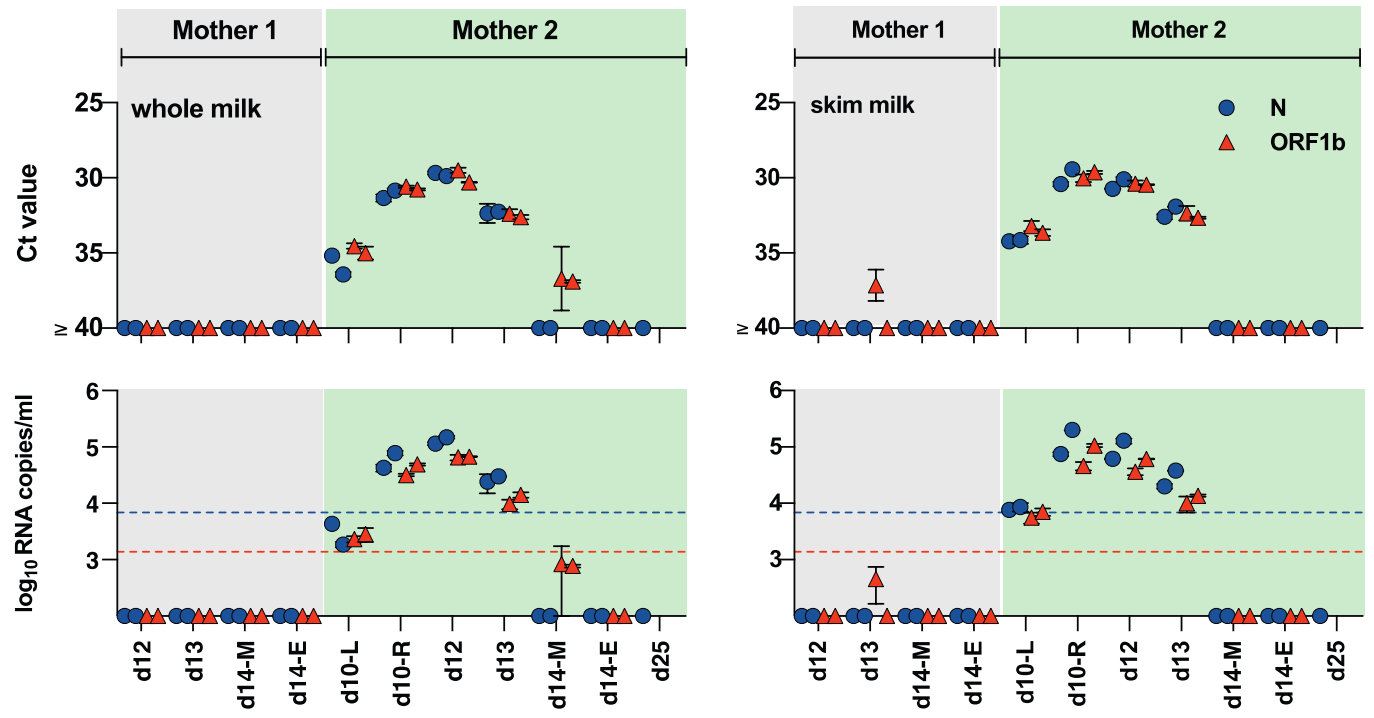

Figure 1. Detection of SARS-CoV-2 in milk of an infected mother. A) Clinical data and time course of infection of both mothers with newborns. After delivery, M1 developed mild COVID-19 symptoms and was subsequently tested positive at day 4 for CoV-2, resulting in spatial isolation of M1 with her newborn (N1). N1 was subsequently tested positive and developed respiratory problems, but both recovered and were discharged 18 days later. M2 was admitted to the same hospital and the same room as M1\&N1. Upon delivery, M2 \& N2 were brought back in the same room until M1 was diagnosed with CoV-2 and isolated. M2 \& N2 were discharged at day 4 but shortly thereafter M2 developed mild COVID-19 symptoms (coughing, sniffing, low fever, loss of taste and smell) and wore since then a surgical mask for 24 hours per day. M2 was tested CoV-2 positive at day 8. At day 11, N2 was tested $\mathrm{CoV}-2$ positive and readmitted to a specialized hospital because of newborn icterus. $\mathrm{N} 2$ then developed severe breathing problems including frequent decreases in oxygen levels requiring ventilation therapy and tube feeding, and then recovered. At day 19, N2 was tested positive for RSV and two days later again for CoV-2. M1 was tested CoV-2 positive again at day 22, 13 days after first being diagnosed with CoV-2. At day 26, M2 \& N2 were tested negative. Dark shading indicates time from first CoV-2 positive swab B) Detection of SARS-CoV-2 RNA in human milk samples by RT-qPCR. RNA isolated from whole and skim milk of different timepoints for both mothers was subjected to analysis by RT-qPCR using primer sets targeting SARS-CoV-2-N and -ORF1b. Samples and viral RNA standard were run in duplicates, isolation and RT-qPCR repeated in two independent assays. RNA from milk of M2 at day 25 was only isolated once and only analyzed by CoV-N RT-qPCR. Symbols at baseline indicate no amplification (or Ct > 36.5 and no amplification in one replicate). Blue dashed line denotes quantification threshold for N (160 copies/rx, Ct 34.2) and red dotted line for ORF1b (32 copies/rx, Ct 35.9). Values below these lines but above baseline indicate amplification in both replicates, but no reliable quantification. Values shown are mean from duplicates \pm SD. Top panel shows Ct values and bottom panel the corresponding viral RNA copy numbers/ml. Abbreviations: d, day; L, left; R, right; M, morning; E, evening 
medRxiv preprint doi: https://doi.org/10.1101/2020.04.28.20075523; this version posted May 4, 2020. The copyright holder for this preprint (which was not certified by peer review) is the author/funder, who has granted medRxiv a license to display the preprint in perpetuity.

All rights reserved. No reuse allowed without permission.

\section{References}

1) Kimberly A Lackey, Ryan M Pace, et al., SARS-CoV-2 and human milk: what is the evidence? medRxiv 2020.04.07.20056812; doi: https://doi.org/10.1101/2020.04.07.20056812

2) Nan Yang, Siyi Che, Jingyi Zhang, et al., Breastfeeding of Infants Born to Mothers with COVID19: A Rapid Review. medRxiv 2020.04.13.20064378; doi:

https://doi.org/10.1101/2020.04.13.20064378

3) Chen L, Li Q, Zheng D, Jiang H, et al., Clinical Characteristics of Pregnant Women with Covid-19 in Wuhan, China. N Engl J Med. 2020 Apr 17.

4) Coronavirus disease (COVID-19) technical guidance: Laboratory testing for 2019-nCoV in humans (WHO), Protocol adapted from "Detection of 2019 novel coronavirus (2019-nCoV) in suspected human cases by RT-PCR" by HKU Med LKS Faculty of Medicine (Poon lab); https://www.who.int/docs/default-source/coronaviruse/peiris-protocol-16-120.pdf?sfvrsn=af1aac73_4 